\title{
EDITORIAL
}

\section{Self-Directed Technology to Improve Urinary Symptoms}

\author{
Joel J. Heidelbaugh, MD, FAAFP, FACG \\ Departments of Family Medicine and Urology, University of Michigan Medical School, Ann Arbor, Michigan
}

Ann Fam Med 2021;19:100-101. https://doi.org/10.1370/afm.2659.

$\mathrm{P}$ rimary care practices commonly encounter women and men of all ages with a wide variety of lower urinary tract symptoms (LUTS) that can negatively impact quality of life. The reported prevalence of stress, urge, or mixed-type urinary incontinence (UI) among women varies widely depending upon population and survey methodology, yet most studies report an average prevalence of $25 \%$ to $45 \%$, and greater than $40 \%$ in women aged over 70 years. ${ }^{1}$ Men primarily seek treatment for LUTS when disruption of daily life (eg, nocturia or storage symptoms including incontinence, frequency, urgency) overcomes potential barriers to treatment. ${ }^{2}$ The self-perception period of LUTS, or watchful waiting period, has been shown to increase relative to severity in a period of 4 to 6 years and is considered a predominant risk factor for benign prostatic hyperplasia $(\mathrm{BPH}){ }^{3}$

Primary care clinics can play an important role in both LUTS and UI management. When patients report urinary symptoms, the first step in care is to assess severity and identify symptoms of stress, urge, or mixed urinary incontinence in both women and men. The American Urological Association Incontinence Symptom Index ${ }^{4}$ is commonly used by urologists, but may be underutilized in primary care. Guidelines emphasize behavioral and pharmacologic strategies to help patients improve symptoms and quality of life. ${ }^{5,6}$ These strategies can be prescribed by primary care doctors before urology referral. Primary

Conflicts of interest: author reports none.

\section{CORRESPONDING AUTHOR}

Joel J. Heidelbaugh

Clinical Professor

Departments of Family Medicine and Urology

University of Michigan Medical School

1018 Fuller St

Ann Arbor, MI 48104

heidel@umich.edu care physicians may choose to prescribe antimuscarinic or anticholinergic medication because it requires less effort than teaching behavioral strategies. Various new e-health programs may help patients to manage their symptoms without medication.

In this issue of Annals of Family Medicine, Loohuis et $\mathrm{al}^{7}$ examine app-based treatment of UI in women, recognizing the myriad of available apps while acknowledging a paucity of evidence for their effectiveness. This study is a randomized controlled trial centered on a novel app that doesn't require caregiver support, and compares the app-based treatment to standard primary care over a period of 4 months. Importantly, the authors conclude that the stand-alone app-based treatment consisting of pelvic floor muscle training and bladder training was nearly equivalent to care-asusual, defined as the aforementioned training, pharmacotherapy, use of a pessary or absorbent products, or referral to a specialist for secondary care. The authors emphasize that "App-based treatment that delivers advice, training, and motivation for managing UI in isolation could offer advantages over care-as-usual, removing the barriers to treatment access and improving adherence to training." ${ }^{17}$ The app may help patients overcome barriers to treatment for UI, such as embarrassment and perception of limited opportunity for symptom improvement, and may support motivated patients. Apps are effective in the self-management of chronic diseases including hypertension and diabetes mellitus, ${ }^{8,9}$ and high-quality apps will without doubt become more prevalent and effective over time for other conditions. This study provides convincing evidence that e-health technology can help patients improve their quality of life with UI.

Albarqouni et $\mathrm{al}^{10}$ conducted a systematic review and meta-analysis examining practices of self-management for men with LUTS, positing that "most men with LUTS can be effectively managed in primary care...with watchful waiting and progressing to drug and surgical interventions if necessary." This is the first systematic review that aims to synthesize the evidence 
for self-directed management interventions to improve LUTS in men. The authors found similar reductions in symptoms, as assessed by the 35 -point International Prostate Symptom Score (IPSS), with self-management as with pharmacotherapeutic interventions for periods of 6 to 12 weeks. They also found that combining self-management with drug interventions at 6 weeks provided additional benefit. One limitation lies in the interpretation and reporting of voiding symptoms in men, yet this is common in trials that survey nonpharmacologic interventions. Another challenge is balancing the presence of symptom with how bothersome they are to the patient, which often does not correlated to patient preference for either behavioral or pharmacologic intervention.

Both of these studies highlight a key opportunity for improving outcomes: patient empowerment through apps and self-management tools. The benefit of both of studies is that patients can in many cases utilize apps and self-management strategies to foster selfcare and yield noticeable improvements in controlling voiding symptoms and UI without the need for pharmacotherapy or surgery. In an era of rapid change in health care delivery, need for cost-effective provisions of care, and evolving use of technology in everyday practice, the advent of app-based and self-management tools holds significant promise when compared with standard care for many common conditions. These strategies will provide enhanced satisfaction for both patients and clinicians.

To read or post commentaries in response to this article, go to https://www.AnnFamMed.org/content/19/2/100/tab-e-letters.
Key words: e-health; self-management; urinary incontinence; lower urinary tract symptoms; family medicine; urology; non-pharmacological intervention

Submitted November 11, 2020; accepted December 1, 2020.

\section{Referencees}

1. Milsom I, Gyhagen M. The prevalence of urinary incontinence. Climacteric. 2019;22(3):217-222.

2. Landau A, Welliver C. Analyzing and characterizing why men seek care for lower urinary tract symptoms. Curr Urol Rep. 2020;21(12):58.

3. Shim SR, Kim JH, Choi H, et al. Association between self-perception period of lower urinary tract symptoms and International Prostate Symptom Score: a propensity score matching study. BMC Urol. 2015;15:30.

4. Scarpero HM, Fiske J, Xue X, Nitti VW. American Urological Association Symptom Index for lower urinary tract symptoms in women: correlation with degree of bother and impact on quality of life. Urology. 2003;61(6):1118-1122.

5. Syan R, Brucker BM. Guideline of guidelines: urinary incontinence. BJU Int. 2016;117(1):20-33.

6. McVary KT, Roehrborn CG, Avins AL, et al. Update on AUA guideline on the management of benign prostatic hyperplasia. J Urol. 2011;185(5):1793-1803.

7. Loohuis AMM, Wessels NJ, Dekker JH, et al. App-Based treatment in primary care for urinary incontinence: a pragmatic, randomized controlled trial. Ann Fam Med. 2021;19(2):102-109.

8. Alessa T, Abdi S, Hawley MS, de Witte L. Mobile apps to support the self-management of hypertension: systematic review of effectiveness, usability, and user satisfaction. JMIR Mhealth Uhealth. 2018;6(7):e10723.

9. Adu MD, Malabu UH, Malau-Aduli AEO, Malau-Aduli BS. Users' preferences and design recommendations to promote engagements with mobile apps for diabetes self-management: Multi-national perspectives. PLoS One. 2018;13(12):e0208942.

10. Albarqouni L, Sanders S, Clark J, et al. Self-management for men with lower urinary tract symptoms: a systematic review and metaanalysis. Ann Fam Med. 2021;19(2):157-167. 